\title{
Confocal Laser Endomicroscopy Can Improve the Diagnosis Rate and Range Assessment of Patients With Conflicting Chronic Atrophic Gastritis Results of White Light Endoscopic and Pathological Diagnosis
}

\section{Suya Pang}

Department of Gastroenterology, Union Hospital, Tongji Medical College, Huazhong University of Science and Technology

\section{Huiying Shi}

Department of Gastroenterology, Union Hospital, Tongji Medical College, Huazhong University of Science and Technology

\section{Hailing Yao}

Department of Gastroenterology, Union Hospital, Tongji Medical College, Huazhong University of Science and Technology

\section{Chen Jiang}

Department of Gastroenterology, Union Hospital, Tongji Medical College, Huazhong University of Science and Technology

\section{Qin Zhang}

Department of Pathology, Union Hospital, Tongji Medical College, Huazhong University of Science and Technology

\section{Rong Lin ( $\square$ selinalin35@hotmail.com )}

Department of Gastroenterology, Union Hospital, Tongji Medical College, Huazhong University of Science and Technology

\section{Research Article}

Keywords: Chronic atrophic gastritis, gastric cancer, white light endoscopy, confocal laser endomicroscopy, pathology

Posted Date: January 6th, 2021

DOI: https://doi.org/10.21203/rs.3.rs-133839/v1 
License: (c) (i) This work is licensed under a Creative Commons Attribution 4.0 International License. Read Full License

Version of Record: A version of this preprint was published at Frontiers in Oncology on March 24th, 2022. See the published version at https://doi.org/10.3389/fonc.2022.809822. 


\section{Abstract}

Background: Chronic atrophic gastritis (CAG) are closely related to the development of gastric cancer. However, the diagnostic accuracy of white light endoscopy (WLE) biopsy for CAG is poor. The diagnostic role and efficacy of confocal laser microscopy (CLE) in CAG missed under WLE biopsy remains unclear.

Methods: This study is a single-center prospective study that included 21 patients who were diagnosed with CAG under WLE, and whose pathological results were chronic inflammation. Then, all these patients received a CLE examination and endoscopic features were evaluated. All patients underwent targeted biopsies and five-point standard biopsies under CLE. The sensitivity, specificity, and accuracy of CLE diagnosis and targeted biopsy were analyzed by using the five-point standard biopsy pathological results.

Results: The pathological results of five-point standard biopsies in 21 patients were CAG, and 17 patients $(81.0 \%)$ were intestinal metaplasia (IM). According to the image diagnosis under CLE, 19 of these 21 patients $(90.5 \%)$ were diagnosed with CAG, and 14 of them (66.7\%) were diagnosed with IM. According to the targeted biopsy under CLE, 17 (81.0\%) patients were diagnosed with CAG, and 14 of them $(66.7 \%)$ were diagnosed with IM. There was no significant difference between CLE image diagnosis and five-point standard biopsies in terms of atrophy severity score $(p=0.927)$, IM severity score $(p=0.250)$, atrophy scope score $(p=0.781)$ and IM scope score $(p=0.195)$. For CAG, the sensitivity and accuracy of CLE image diagnosis were higher than those of CLE targeted biopsies ( $90.5 \%$ vs. $81.0 \%, p=0.331)$. For IM, the sensitivity, specificity and accuracy of CLE image diagnosis were the same as those of CLE targeted biopsies.

Conclusions: CLE can improve the diagnosis rate of CAG, and can increase the comprehensive assessment of the scope and severity of CAG.

\section{Introduction}

As the fourth most common cancer worldwide, gastric cancer has great significance in prevention and treatment ${ }^{1}$. Chronic atrophic gastritis (CAG) is a well-recognized precancerous lesion of gastric cancer that its diagnosis and follow-up is critical ${ }^{2,3}$. The most common diagnostic and follow-up method of CAG currently is white light endoscopy (WLE) and endoscopic biopsy.

However, WLE cannot accurately diagnose CAG, and the WLE diagnosis has two limitations. Firstly, WLE cannot accurately locate the lesion, resulting in failure to target biopsy. Therefore, there may be inconsistencies between WLE diagnosis and pathological diagnosis ${ }^{4}$. Pan et al. reported that the coincidence rate of WLE and pathological diagnosis of CAG was only $50.32 \%{ }^{5}$. Secondly, local biopsy under WLE not only cannot accurately reflect the severity of the lesion because it cannot achieve targeted biopsy, but also cannot assess the extent of the lesion. However, the degree and scope of atrophy affect its prognosis ${ }^{6,7}$. 
Confocal laser endomicroscopy (CLE) stand at the forefront of the novel endoscopic techniques. CLE permits direct in vivo identification and clear visualization of gastric patterns at the cellular level ${ }^{8,9}$, which makes it assess the severity and scope of atrophy effectively. Several studies reported that the CLE is useful in the diagnosis of cancer and premalignant lesions ${ }^{10-12}$. Furthermore, some studies showed that the CLE had greater consistency with the pathological diagnosis result than the WLE ${ }^{13,14}$.

Actually, a great part of patients had the CAG diagnosis with WLE and the chronic inflammation diagnosis with histopathology clinically ${ }^{4}$, which brings difficulty in final diagnosis and treatment for clinicians. Therefore, in this study we aim to use the CLE to diagnose those patients who were diagnosed with CAG under WLE, and whose pathological results were chronic inflammation, to explore the diagnostic value of CLE for CAG patients.

\section{Methods}

\section{Patients}

A total of 21 patients enrolled in this study were from the Union Hospital of Tongji Medical College of Huazhong University of Science and Technology according to the inclusion and exclusion criteria. Inclusion criteria were: (i) 18-80 years old, can endure digestive endoscopy; (ii) patients with WLE diagnosis of CAG and pathological results were chronic inflammation in nearly 3 months; (iii) voluntarily join the experiment and sign a written informed consent. Exclusion criteria were as follows: (i) patients with gastrectomy history, acute gastrointestinal bleeding history, advanced gastric cancer and other gastrointestinal malignancies; (ii) patients suffering from disease cannot tolerate gastroscopy, like coagulation dysfunction, renal insufficiency, serious cardiopulmonary disease and sodium fluorescein allergy; (iii) pregnant and lactating women; (iv) no legal capacity or having medical and ethical reasons affect the study continues. All patients included in this study signed informed consent.

\section{CLE procedure}

All patients were given oral saline solution containing simethicone emulsion (Berlin-Chemie AG) to remove excess mucus from gastric mucosa before examination. And all patients undergo a skin test of fluorescein sodium (Baiyunshan Mingxing Pharmaceutical Co. Ltd., Guangzhou, China) before the examination to ensure that allergic reactions do not occur, which was used as a contrast agent intravenously before CLE.

First observe the condition of the mucosa through WLE, select erosion, elevated, hyperemia, rough or granular mucosa, and gray intestinal-type epithelium as target then using CLE to observe ${ }^{15,16}$. CLE (YZB/FRA 5399 - 2012) was performed by an experienced endoscopist and an assistant. Five standardized intragastric sites were observed on CLE: lesser curvature of the antrum and greater curvature of the antrum, stomach angle, lesser curvature of the corpus and greater curvature of the corpus. Meanwhile 5 to $10 \mathrm{CLE}$ images of different mucosal depths and CLE video were collected from each site, and the images and videos were stored for later assessment. Endoscopist performed real-time 
diagnosis, targeted biopsy and five standard site biopsies under CLE. And another experienced endoscopist diagnosed through images and videos and was blinded to the basic information and medical history of patients.

The standard criteria for endoscopists to diagnose CAG with CLE is that stomach pits are sparse, interstitial widening, irregular arrangement, severely reduced number of gastric pits, dilated openings, decreased number of subepithelial capillaries ${ }^{17}$. The criteria for diagnosing IM with CLE are goblet cells, villous-like pits, absorptive cells and brush borders ${ }^{18}$. (Fig. 1)

\section{Histopathology}

Mucosal biopsy specimens were placed in $4 \%$ methanal and sectioned into $4 \mu m$ thickness, and stained with hematoxylin and eosin (HE). An experienced pathologist reviewed all the biopsy specimens who was blinded to the results of the endoscopic findings and the medical history of patients. CAG was recognized morphologically by mild epithelial abnormalities, including a decrease in the number of cytoplasmic mucins, an increase in the nucleus and nucleolus, and an increase in mitotic figures in the pits of the stomach, when inflammation is extensive and accompanied by glandular atrophy ${ }^{19}$. And the CAG was further divided into mild, moderate and severe by a rough assessment of the relationship between the thickness of the gland and the thickness of the entire mucosa. IM was recognized morphologically by goblet cells and absorptive cells, and was further divided into mild, moderate and severe according to the number of abnormal cells ${ }^{20}$. (Fig. 1)

\section{Study definitions}

Atrophy range score was a diagnosis of CLE and five standard site biopsy pathologies for the scope of atrophy. The diagnosis results of different parts of the stomach with or without atrophy were recorded as 1 point and 0 point respectively. Atrophy severe score was a diagnosis to evaluate the severity of atrophy by CLE and five standard site biopsy pathologies. Diagnosis of different parts of the stomach as mild to moderate atrophy was recorded as 1 point, 2 points and 3 points, respectively. IM range score and IM severe score were defined in the same way.

\section{Statistical analysis}

Data were entered into Excel data sheets and analyzed with SPSS software v25.0 (IBM, USA) or SAS software, version 9.4. Clinical characteristics were expressed as median and range, absolute value or fractions. Patients characteristics were compared using X2 or Fisher exact for categorical variables. A Mann-Whitney U-test was used to compare the median duration between groups. $\mathrm{P}<0.05$ was considered statistically significant.

\section{Results}

\section{Patient characteristics}


We included 21 patients with WLE diagnosis of CAG and chronic inflammation on pathological examination during nearly 3 months in this prospective study. The overall median age was 54 years (range: 27-66 years), with the ratio of female to male 1.33. 14 (66.7\%) patients had a history of proton pump inhibitors (PPI) medication. 8 (38.1\%) patients have a history of smoking and drinking. And 8 (38.1\%) patients were anxious and $3(14.3 \%)$ patients were depressed. The demographic characteristics of patients identified in this study were summarized in Table 1.

Table 1

Patient demographics and clinical features in this study.

\begin{tabular}{|ll|}
\hline Characteristics & All patients $(\mathbf{n = 2 1 , \% )}$ \\
\hline Mean age, $y$ (range) & $54(27-66)$ \\
\hline Male & $9(42.9)$ \\
Female & $12(57.1)$ \\
History of PPI use & $14(66.7)$ \\
Smoking habits & $8(38.1)$ \\
\hline Drinking habits & $8(38.1)$ \\
Anxiety & $8(38.1)$ \\
\hline Depression & $3(14.3)$ \\
\hline
\end{tabular}

\section{CLE results and Pathological diagnosis results}

Of the 21 patients, pathological results of five standard site biopsies all showed CAG, and $17(81.0 \%)$ of them had IM. The real-time diagnosis results through CLE and the post-diagnosis results through images and videos were consistent. Of the 21 patients, 19 (90.5\%) patients were diagnosed with CAG by CLE, and $14(66.7 \%)$ of them were diagnosed with IM. In the case of targeted biopsy, 17 (81.0\%) patients were diagnosed with CAG, 14 (66.7\%) of whom had IM.

Most patients had CAG and IM in multiple sites. Compared with targeted biopsy, CLE image diagnosis was more consistent with five-point standard biopsies in diagnosis of CAG and IM. There was no significant difference between the CLE diagnosis and the pathological diagnosis of five standard site biopsies for the atrophy range score and atrophy severe score $(p=0.781 ; p=0.927)$, and also for the IM range score and IM severe score $(p=0.195 ; p=0.250)$. The results of confocal diagnosis, targeted biopsy and five standard site biopsies were shown in Table 2 . 
Table 2

The diagnosis results of CLE image diagnosis, CLE targeted biopsies and five-point standard biopsies.

\begin{tabular}{|c|c|c|c|c|c|}
\hline & CLE diagnosis & Targeted biopsy & Five standard site biopsies & $P$ & $P$ \\
\hline No-CAG/IM & 2 & 5 & 0 & 0.488 & 0.027 \\
\hline CAG & 19 & 17 & 21 & 0.488 & 0.027 \\
\hline antrum & 3 & 9 & 5 & 0.697 & 0.012 \\
\hline angle & 3 & 2 & 0 & 0.106 & 0.139 \\
\hline body & 0 & 0 & 1 & 1.000 & 0.618 \\
\hline multiple parts & 13 & 6 & 15 & 0.731 & 0.026 \\
\hline \multicolumn{6}{|l|}{ Atrophy score } \\
\hline range & 46 & - & 48 & 0.781 & - \\
\hline severe & 80 & - & 79 & 0.927 & - \\
\hline IM & 14 & 14 & 17 & 0.484 & 0.242 \\
\hline antrum & 1 & 4 & 3 & 0.607 & 0.077 \\
\hline angle & 2 & 0 & 2 & 1.000 & 0.493 \\
\hline body & 0 & 0 & 0 & - & - \\
\hline multiple parts & 11 & 10 & 12 & 0.698 & 0.637 \\
\hline \multicolumn{6}{|l|}{ IM score } \\
\hline range & 33 & - & 42 & 0.195 & - \\
\hline severe & 64 & - & 76 & 0.250 & - \\
\hline
\end{tabular}

The sensitivity and accuracy of CLE image in the diagnosis of CAG were both $90.5 \%$, and of CLE targeted biopsy were both $81.0 \%$. For CAG, CLE image diagnosis had higher sensitivity and accuracy than CLE targeted biopsies, but not statistically significant $(p=0.331)$, but for $I M$, they were same. The sensitivity of CLE image and CLE targeted biopsy in the diagnosis of IM was both $76.5 \%$, the specificity was $75 \%$, and the accuracy was $76.2 \%$. The results of diagnostic efficiency of CLE and targeted biopsy on CAG and IM were shown in Table 3. 
Table 3

Diagnostic efficiency of CLE and targeted biopsy on CAG and IM.

\begin{tabular}{|lllllll|}
\hline \multicolumn{5}{c}{ CAG } & \multicolumn{3}{c|}{ IM } \\
\hline & Sensitivity & Specificity & Accuracy & Sensitivity & Specificity & Accuracy \\
\hline CLE & $90.5 \%$ & - & $90.5 \%$ & $76.5 \%$ & $75 \%$ & $76.2 \%$ \\
\hline Targeted biopsy & $81.0 \%$ & - & $81.0 \%$ & $76.5 \%$ & $75 \%$ & $76.2 \%$ \\
\hline$p$ & 0.331 & - & 0.331 & 0.672 & 0.672 & 0.672 \\
\hline
\end{tabular}

\section{Discussion}

Each year there were more than 900,000 new diagnoses of gastric cancer worldwide and gastric cancer was the second and fourth most common cause of cancer death in males and females, respectively. ${ }^{21}$ As a precancerous lesion of gastric cancer, CAG is of great significance in diagnosis. There was a great part of patients had inconsistent results of WLE and pathological diagnosis of CAG clinically ${ }^{4}$, which made difficulties in clinical diagnosis and treatment. But there were seldom studies for the diagnosis of these contradictory patients. CLE can realize real-time diagnosis at the cellular level and a larger evaluated area in vivo identification compared to biopsies ${ }^{8,9}$. And many studies have shown that CLE has a high diagnostic value for precancerous lesions ${ }^{22,23}$, but seldom studies had used CLE to diagnose patients with inconsistent WLE diagnosis and pathology diagnosis. Therefore, this study made a prospective study of these patients with conflicting diagnosis results, and made diagnosis of CAG and IM by CLE, targeted biopsy and five standard site biopsies which was regarded as the gold standard, so as to evaluate the diagnostic efficacy of CLE for CAG patients.

We studied 21 patients with conflicting diagnosis results, then all patients were diagnosed as CAG by the gold standard, and $90.5 \%$ of patients were diagnosed with CAG through CLE. The sensitivity of CLE diagnosis of atrophy in the study was as high as in other studies (range from $85-90 \%)^{24-33}$. Besides, targeted biopsy under CLE also diagnosed $81.0 \%$ of patients with CAG, which indicated that CLE can improve biopsy accuracy. As a result, CLE can improve the diagnosis rate of CAG. However, the accuracy of CAG diagnosis by targeted biopsy was lower than that of CLE. This may be because the biopsy under CLE can only be performed after the confocal probe was removed, and there may be displacement during this process. If there is a device that can simultaneously biopsy in the CLE magnified field of view, this problem may be solved. What is more, CLE did not differ significantly from the gold standard in assessing the scope and severity of atrophic and IM. This shows that CLE can achieve a comprehensive assessment of the scope and severity of CAG and IM, compared with WLE.

In conclusion, CLE can solve the contradiction between WLE and pathological diagnosis, and increased the diagnostic rate of patients with different WLE and pathological diagnosis. CLE can achieve targeted biopsy, and as a real-time pathology, the diagnostic efficiency was even higher than that of targeted 
biopsy. The most important point was that CLE had a good advantage in the diagnosis, follow-up and comprehensive evaluation of CAG, and it was significantly better than WLE and biopsy under WLE. CLE had good clinical significance for the diagnosis of CAG.

\section{Abbreviations}

CAG: Chronic atrophic gastritis; WLE: white light endoscopy; CLE: confocal laser microscopy; IM: intestinal metaplasia.

\section{Declarations}

\section{Ethics approval and consent to participate}

The study was approved by the Ethics Committee of Tongji Medical College, Huazhong University of Science and Technology (IORG No: IORG0003571), and performed in accordance with the Declaration of Helsinki.

\section{Consent for publication}

Not Applicable.

\section{Availability of data and materials}

The datasets used and/or analysed during the current study are available from the corresponding author on reasonable request.

\section{Competing interests}

The authors declare that they have no competing interests.

\section{Funding}

This study was supported by the National Natural Science Foundation of China (nos. 81770539, 81974068, 81900580), the Natural Science Foundation of Hubei Province (no. 2017CFA061) and the National Key Research and Development program of China (no. 2017YFC0110003).

\section{Authors' contributions}

RL designed and supervised the study and data analysis. SYP and HYS performed most of the investigation, data analysis and wrote the manuscript; $\mathrm{HLY}$ and $\mathrm{CJ}$ contributed to interpretation of the data and analyses; QZ provided pathological diagnosis assistance.

\section{Acknowledgments}


We are very grateful to the units and individuals who have given us support and help, especially the Digestive Endoscopy Center of Union Hospital affiliated to Tongji Medical College of Huazhong University of Science and Technology, which has provided great support for this study.

\section{References}

1. Kamangar F, Dores GM, Anderson WF. Patterns of cancer incidence, mortality, and prevalence across five continents: defining priorities to reduce cancer disparities in different geographic regions of the world. J Clin Oncol 2006; 24:2137-2150.

2. Islami F, Sheikhattari P, Ren JS, Kamangar F. Gastric atrophy and risk of oesophageal cancer and gastric cardia adenocarcinoma-a systematic review and meta-analysis. Ann Oncol. 2011; 22: 754760.

3. Weck MN, Brenner H. Prevalence of chronic atrophic gastritis in different parts of the world. Cancer Epidemiol Biomarkers Prev 2006; 15:1083-94.

4. Yu X, Chen J, Zhang L, et al. Quantitative Diagnosis of Atrophic Gastritis by Probe-Based Confocal Laser Endomicroscopy. Biomed Res Int.2020 Mar 2; 2020:9847591.

5. Pan D, Sun SY, Liu X, et al. Comparison of chronic atrophic gastritis by endoscopy and the pathological results. Journal of Modern Oncology, 2018,26(06):899-902.

6. den Hoed CM, Holster IL, Capelle LG, et al. Follow-up of premalignant lesions in patients at risk for progression to gastric cancer. Endoscopy. 2013;45(4):249-256. doi:10.1055/s-0032-1326379.

7. Correa P, Piazuelo MB. The gastric precancerous cascade.J Dig Dis. 2012;13(1):2-9. doi:10.1111/j.1751-2980.2011.00550.x.

8. Dunbar KB. Endomicroscopy in Barrett's esophagus. Gastrointest Endosc Clin N Am 2013; 23:56579.

9. Wallace MB, Sharma P, Lightdale C, Wolfsen H, Coron E, Buchner A, et al. Preliminary accuracy and interobserver agreement for the detection of intraepithelial neoplasia in Barrett's esophagus with probe-based confocal laser endomicroscopy. Gastrointest Endosc 2010; 72:19-24.

10. Li W.-B., Zuo X.-L., Zuo F., et al. Characterization and identification of gastric hyperplastic polyps and adenomas by confocal laser endomicroscopy. Surgical Endoscopy. 2010;24(3):517-524.

11. Banno K., Niwa Y., Miyahara R., et al. Confocal endomicroscopy for phenotypic diagnosis of gastric cancer. Journal of Gastroenterology and Hepatology. 2010;25(4):712-718.

12. Li W.-B., Zuo X.-L., Li C.-Q., et al. Diagnostic value of confocal laser endomicroscopy for gastric superficial cancerous lesions. Gut. 2011;60(3):299-306.

13. Hoffman A, Manner H, Rey JW, Kiesslich R. A guide to multimodal endoscopy imaging for gastrointestinal malignancy - an early indicator. Nat Rev Gastroenterol Hepatol. 2017; 14:421-434.

14. Li W.-B., Zuo X.-L., Li C.-Q., et al. Diagnostic value of confocal laser endomicroscopy for gastric superficial cancerous lesions. Gut. 2011;60(3):299-306. 
15. Kimura K., Takemoto T. An endoscopic recognition of the atrophic border and its significance in chronic gastritis. Endoscopy. 1969;1(3):87-97.

16. Dai Y.-C., Tang Z. P., Zhang Y. L. How to assess the severity of atrophic gastritis. World Journal of Gastroenterology. 2011;17(13):1690-1693.

17. Liu T., Zheng H., Gong W., Chen C., Jiang B. The accuracy of confocal laser endomicroscopy, narrow band imaging, and chromoendoscopy for the detection of atrophic gastritis. Journal of Clinical Gastroenterology. 2015;49(5):379-386.

18. Bai T, Zhang L, Sharma S, et al. : Diagnostic performance of confocal laser endomicroscopy for atrophy and gastric intestinal metaplasia: A meta-analysis.J Dig Dis.2017;18(5):273-82.

19. Dixon M. F., Genta R. M., Yardley J. H., Correa P. Classification and grading of gastritis: the updated Sydney system. The American Journal of Surgical Pathology. 1996;20(10):1161-1181.

20. Ji R, Zuo XL, Yu T, Gu XM, Li Z, Zhou CJ, Li YQ. Mucosal barrier defects in gastric intestinal metaplasia: in vivo evaluation by confocal endomicroscopy. Gastrointest Endosc. 2012;75:980-987.

21. D. Forman, V.J. BurleyGastric cancer: global pattern of the disease and an overview of environmental risk factors. Best Pract Res Clin Gastroenterol, 20 (2006), pp. 633-649

22. Kollar M, Spicak J, Honsova E, Krajciova J, Vackova Z, Martinek J (2018) Role of confocal laser endomicroscopy in patients with early esophageal neoplasia. Minerva Chir 73(4):417-427.

23. Zhang HP, Yang S, Chen WH, et al. The diagnostic value of confocal laser endomicroscopy for gastric cancer and precancerous lesions among Asian population: a system review and metaanalysis. Scand J Gastroenterol. 2017;52(4):382-8.

24. Zhang JN, Li YQ, Zhao YA et al. Classification of gastric pit patterns by confocal endomicroscopy. Gastrointest Endosc 2008; 67: 843-53.

25. Wang P, Ji R, Yu T et al. Classification of histological severity of Helicobacter pylori-associated gastritis by confocal laser endomicroscopy. World J Gastroenterol 2010; 16: 5203-10.

26. Li Z, Zuo XL, Yu T et al. Confocal laser endomicroscopy for in vivo diagnosis of gastric intestinal metaplasia: a randomized controlled trial. Endoscopy 2014; 46: 282-90.

27. Lim LG, Yeoh KG, Salto-Tellez M et al. Experienced versus inexperienced confocal endoscopists in the diagnosis of gastric adenocarcinoma and intestinal metaplasia on confocal images. Gastrointest Endosc 2011; 73: 1141-7.

28. Li WB, Zuo XL, Li CQ et al. Diagnostic value of confocal laser endomicroscopy for gastric superficial cancerous lesions. Gut 2011; 60: 299-306.

29. Guo YT, Li YQ, Yu T et al. Diagnosis of gastric intestinal metaplasia with confocal laser endomicroscopy in vivo: a prospective study. Endoscopy 2008; 40: 547-53.

30. Lim LG, Yeoh KG, Srivastava S, Chan YH, Teh M, Ho KY. Comparison of probe-based confocal endomicroscopy with virtual chromoendoscopy and white-light endoscopy for diagnosis of gastric intestinal metaplasia. Surg Endosc 2013; 27: 4649-55. 
31. Pittayanon R, Rerknimitr $\mathrm{R}$, Wisedopas $\mathrm{N}$ et al. Flexible spectral imaging color enhancement plus probe-based confocal laser endomicroscopy for gastric intestinal metaplasia detection. J Gastroenterol Hepatol 2013; 28: 1004-9.

32. Li Z, Zuo XL, Li CQ et al. New classification of gastric pit patterns and vessel architecture using probe-based confocal laser endomicroscopy. J Clin Gastroenterol 2016; 50: 23-32.

33. Liu T, Zheng H, Gong W, Chen C, Jiang B. The accuracy of confocal laser endomicroscopy, narrow band imaging, and chromoendoscopy for the diagnosis of atrophic gastritis. J Clin Gastroenterol 2015; 49: 379-86.

\section{Figures}



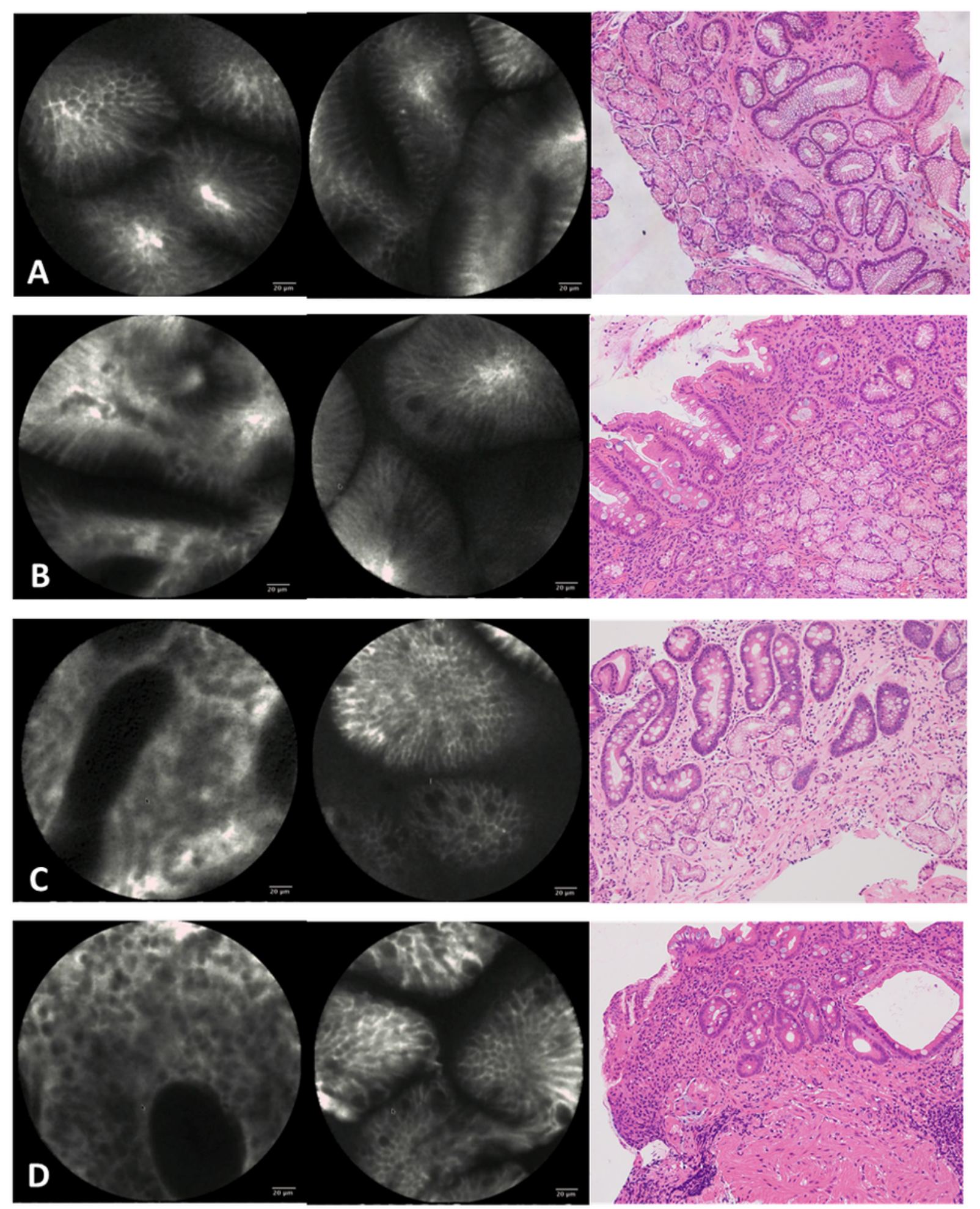

\section{Figure 1}

Confocal imaging and histopathology of gastric epithelium. A, Normal gastric epithelium. B, Mild atrophic gastritis and intestinal metaplasia. C, Moderate atrophic gastritis and intestinal metaplasia. D, Severe atrophic gastritis and intestinal metaplasia. 